\title{
Übersichten
}

Z Gerontol Geriat 2021 · 54:695-703 https://doi.org/10.1007/s00391-020-01732-3 Eingegangen: 11. Dezember 2019

Angenommen: 7. April 2020

Online publiziert: 7. Mai 2020

(c) Der/die Autor(en) 2020

F. Schumacher-Schönert ${ }^{1} \cdot$ D. Wucherer ${ }^{1} \cdot$ A. Nikelski ${ }^{3} \cdot$ S. Kreisel $^{3} \cdot$ H. C. Vollmar ${ }^{4}$. W. Hoffmann ${ }^{1,2} \cdot$ J. R. Thyrian ${ }^{1,2}$

${ }^{1}$ AG Interventionelle Versorgungsforschung, Deutsches Zentrum für Neurodegenerative Erkrankungen e. V. (DZNE) Standort Rostock/Greifswald, Greifswald, Deutschland

${ }^{2}$ Institut für Community Medicine, Abteilung Versorgungsepidemiologie und Community Health, Universitätsmedizin Greifswald, Greifswald, Deutschland

${ }^{3}$ Evangelisches Klinikum Bethel gGmbH, Akademisches Lehrkrankenhaus, Universität Münster, Münster, Deutschland

${ }^{4}$ Abteilung für Allgemeinmedizin, Ruhr-Universität Bochum, Bochum, Deutschland

\section{Das Entlassmanagement deutscher Krankenhäuser für kognitiv beeinträchtigte, ältere Menschen - ein Scoping Review}

enten vorbereiten [18]. Darüber hinaus steht nach der Entlassung aus dem Krankenhaus oft keine professionelle Kontaktperson zur Verfügung, und die medizinische Nachsorge (z.B. eine fachärztliche Anschlussbehandlung) muss von den MmkB selbst oder - sofern vorhanden - von deren pflegenden Angehörigen organisiert werden [11, 21]. Versorgungslücken können zu einer vorzeitigen Institutionalisierung führen [10], erhöhen ungeplante Wiedereinweisungen ins Krankenhaus [12] und das Sterberisiko signifikant [29]. Krankenhausentlassungen von MmkB sollten so geplant werden, dass die Rückkehr in die Häuslichkeit erleichtert wird $[11,19]$. Dabei ist es besonders wichtig, die Überleitung von MmkB vom Krankenhaus in die Häuslichkeit möglichst bruchlos und bedarfsgerecht zu organisieren [2].

In den letzten Jahren wurden sehr viele Bemühungen um das Entlassmanagement (EM) deutscher Krankenhäuser unternommen; unter anderem wurden Expertenstandards definiert [28] und in 2017 wurde das EM für Krankenhäuser gesetzlich verpflichtend im SGB V $₫ 39$ Absatz 1a verankert [8]. Obwohl durch die gesetzliche Verankerung eine gewisse Homogenität hergestellt werden konnte, fehlt es übergreifend an einheitlichen Standards. Evaluierte (Begleit-)Konzepte zum Entlassmanagement bei MmkB sind rar. Modellprojekte der Pflegeüberleitung ab 2007 liegen z. B. von Wingenfeld et al. vor [30].

\section{Zielsetzung}

Die Arbeit soll den aktuellen Stand der Wissenschaft und die vorhandene Evidenzzum Entlassmanagement bei MmkB oder MmD darstellen. Zusätzlich werden Angaben zur Notwendigkeit eines speziellen Managements für MmkB und $\mathrm{MmD}$ über die Sektorengrenzen daraus hergeleitet.

\section{Methoden}

Mithilfe eines Scoping Review wurden alle relevanten, empirischen Arbeiten und Veröffentlichungen zu Studien und Projekten von $\mathrm{MmkB}$ und $\mathrm{MmD}$ in Deutschland an der Schnittstelle vom Krankenhaus zurück in die Häuslichkeit identifiziert und eine explorative Literaturanalyse durchgeführt.

\section{Suchstrategie}

Die Suche erfolgte vom Februar bis zum Mai 2019 in PubMed (Zusatzmaterial online: Anlage 1). gen können sich dementsprechend unzureichend auf die Entlassung des Pati- 
102 für das Screening

identifizierte Arbeiten

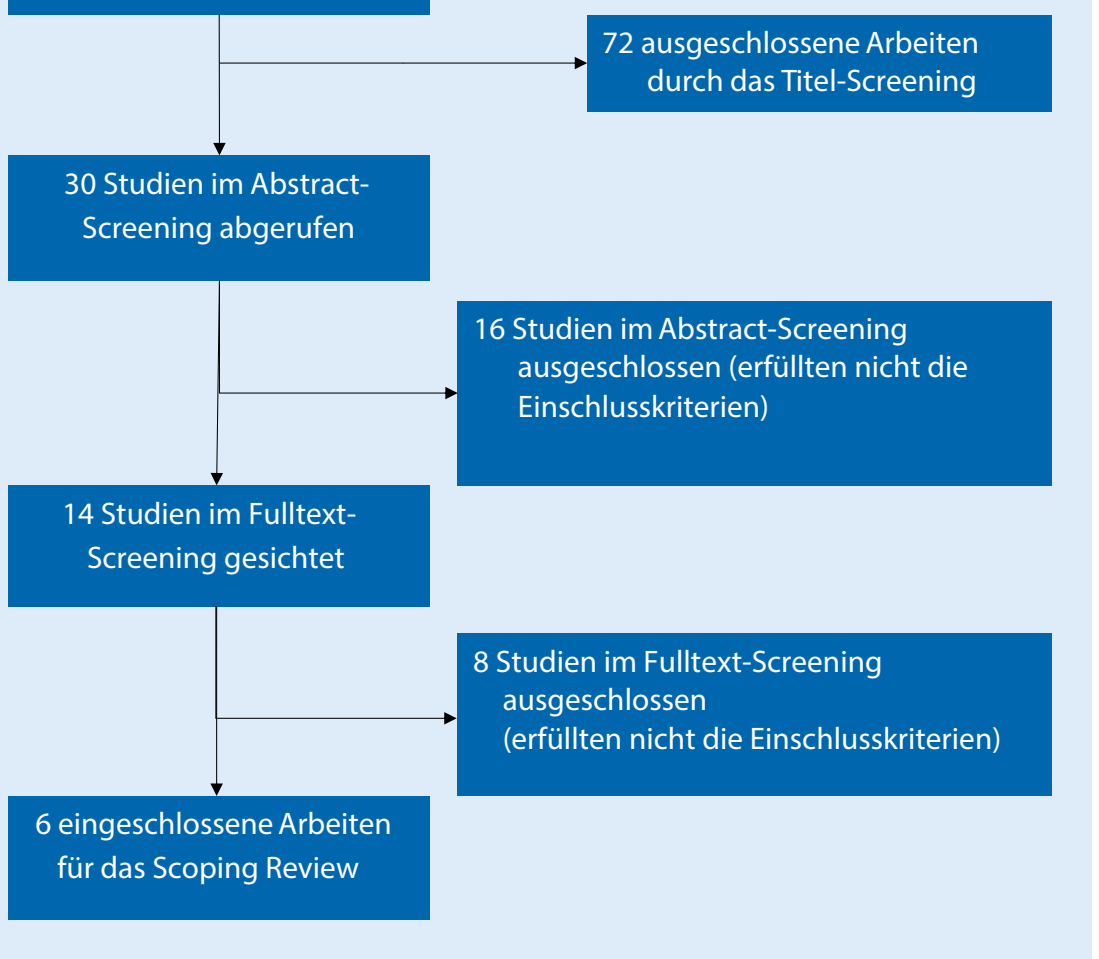

Abb. 1 - Screeningflussdiagramm. Studien wurden nicht ohne weitere Prüfung abgelehnt, wenn sie bereits eines der Ausschlusskriterien erfüllten, sondern ggf. nochmals gesichtet, um Angaben zu Prozess-, Struktur- und Ergebnisqualität der Studiensettings ausfindig zu machen

Als finale Suchleitwörter wurden "(hospital OR acute care) AND (dementia OR cognitive impairment OR Alzheimer's disease) AND (discharge OR transition) AND Germany"verwendet. Die Begriffe wurden in Anlehnung an das PIKOModell [27] definiert:

- Population - Menschen mit kognitiven Beeinträchtigungen oder mit Demenz in deutschen Krankenhäusern,

- Intervention - strukturiertes Überleitungs- und/oder Entlassmanagement,

- Kontrollgruppe - keine,

- Outcome - patientenzentrierte Outcomes, Angaben zu Krankenhauswiedereinweisungen, Struktur-, Prozessund Ergebnisqualität.

Es wurden 102 Ergebnisse in der wissenschaftlichen Literaturdatenbank PubMed identifiziert und einem Titelscreening unterzogen. Dreißig Artikel wurden für das darauffolgende "abstract screening“ herangezogen. Aus Titel- und Abstract screening wurden Artikel ausgeschlossen, die bereits dem Titel nach aufgrund ihres Kernthemas nicht relevant schienen; auf dieser Stufe wurden Studien zur Grundlagenforschung $(n=19)$, zu Krebs-, Herz-, Kreislauf-, der Transplantations- und Adoleszenzforschung ausgeschlossen. In dem anschließenden "full text screening" wurden Artikel mit Kernthemen $\mathrm{zu}(\mathrm{r})$ geriatrischen Patienten, demenzsensibler Pflege im Krankenhaus, kognitiv beeinträchtigten Menschen ohne Bezug auf das Entlassmanagement und Artikel mit direktem Bezug zu einem Entlassmanagement im Akutkrankenhaus herangezogen. Im weiteren Verlauf des Full text screening wurden weitere 6 Artikel ausgeschlossen, da diese sich im Kern mit den Themen der Angehörigenbelastung $(n=2)$, der Veränderung des kognitiven Status während eines rehabilitativen Aufenthaltes $(n=2)$, der Vorhersage von Demenzen mittels kognitivem Screening $(n=2)$ sowie mit einem Vergleich zwischen 2 nichtakutstationären Settings (,inpatient" vs. „day clinic treatment“; $n=1$ ) auseinandersetzten.

\section{Ein- und Ausschlusskriterien}

In die Übersichtsarbeit wurden Studien und Arbeiten eingeschlossen, die sich inhaltlich mit dem Thema des Entlassmanagements deutscher Krankenhäuser im Zusammenhang mit der Versorgung kognitiv beeinträchtigter Menschen auseinandersetzten. Dabei wurden die Arbeiten durch die Autorin in 3 Kategorien eingeordnet:

a) eine Information zum Entlassmanagement im Krankenhaus ist vorhanden,

b) eine Information zum Entlassmanagement im Krankenhaus unter Berücksichtigung der speziellen Pflege von MmkB und MmD wird dargestellt,

c) ein Bezug zur gesetzlichen Verpflichtung eines standardisierten Entlassmanagement nach $₫ 39$ (1a) SGB V seit Oktober 2017 wurde hergestellt.

\section{Datenextraktion}

Für jede Studie wurden die Grunddaten, einschließlich Autoren, Erscheinungsjahr und Studienregion, erfasst. Ergänzend wurden Informationen zur Studienart, zum Setting, der Studienfokus, zu den Interventions- und OutcomeParametern und die Gütekriterien der Qualitätsbewertung erfasst.

Darüber hinaus wurden patientenzentrierte Outcomes, Interventionseffekte, Gemeinsamkeiten - Unterschiede sowie die innovativen Ansätze der Studien herausgearbeitet.

Im Rahmen des Vorgehens wurden im Verlauf der Übersichtsarbeit ergänzend und - sofern vorhanden - Informationen zu Struktur-, Prozess- und Ergebnisqualität der Krankenhäuser und des Studienvorgehens abstrahiert. 


\section{Datenanalyse}

\section{Qualitätsbewertung der eingeschlossenen Studien}

Die Qualitätsbewertung der eingeschlossenen Studien erfolgte über das Qualitätskriterienblatt (Zusatzmaterial online: Anlage 2) basierend auf dem Mixed Methods Appraisal Tool (MMAT). Die Bewertung der Gütekriterien erfolgte über die Leitfragen zu Objektivität, Reliabilität und Validität. Die Übersichtsarbeit wurde insgesamt unter Beachtung des PRISMA Statement für Scoping Reviews und dessen Checkliste der Berichterstattung erstellt [15]. Neben der Autorin (F. Schumacher-Schönert) sichtete ein weiterer Gutachter (Reviewer, J.R. Thyrian) die ausgewählten Artikel und bewertete diese unabhängig von der Autorin.

\section{Ergebnisse}

\section{Studienauswahl}

Das Flussdiagramm des Screeningprozesses ist in - Abb. 1 dargestellt.

Die in Tab. 1 und 2 dargestellten 6 Artikel erfüllten die Einschlusskriterien der vorliegenden Arbeit.

\section{Bezug zum Entlassmanagement}

Eine Information zum generellen Entlassmanagement im Krankenhaus wurde in 3 der 6 eingeschlossenen Arbeiten gegeben, Informationen zum speziellen Entlassmanagement für MmkB und $\mathrm{MmD}$ waren in 5 von 6 Arbeiten enthalten. Alle eingeschlossenen Arbeiten erschienen mindestens 2 Jahre vor der gesetzlichen Verpflichtung eines standardisierten Entlassmanagement seit Oktober 2017 und konnten somit keinen Bezug dazu darstellen.

Angerhausen [2] fokussierte das prozessuale, ganzheitliche Denken und Handeln im interdisziplinären Arbeitsprozess des Entlassmanagements. Im Rahmen des Entlassmanagements wurde vielfach das Medikationsmanagement und/oder die Verringerung der Überleitung in eine institutionalisierte Pflege verstanden.

Z Gerontol Geriat 2021 · 54:695-703 https://doi.org/10.1007/s00391-020-01732-3

(c) Der/die Autor(en) 2020

F. Schumacher-Schönert · D. Wucherer · A. Nikelski · S. Kreisel · H. C. Vollmar · W. Hoffmann . J. R. Thyrian

\section{Das Entlassmanagement deutscher Krankenhäuser für kognitiv beeinträchtigte, ältere Menschen - ein Scoping Review}

\section{Zusammenfassung}

Hintergrund. In deutschen Krankenhäusern sind etwa $40 \%$ aller Patienten über 65 Jahre kognitiv beeinträchtigt. Für diese ist es besonders wichtig, dass die Überleitung in die Häuslichkeit möglichst bruchlos und vollumfänglich bedarfsgerecht organisiert ist. Ziel der Arbeit. Ziel der Arbeit ist es, einen systematischen Überblick über Evidenz des Entlassmanagements (EM) bei Menschen mit kognitiven Beeinträchtigungen (MmkB) oder Demenz (MmD) zu geben. Ferner soll geprüft werden, ob sich, darauf aufbauend, die Notwendigkeit eines sektorenübergreifenden Konzeptes ergibt.

Material und Methoden. Anhand eines Scoping Review wurden $n=102$ Publikationen identifiziert, von denen $n=6$ in die Analysen eingingen.

Ergebnisse. Der Artikel gibt eine Übersicht über die aktuelle Versorgung von MmkB in deutschen Akutkrankenhäusern. Generelle
Informationen zum EM im Krankenhaus wurden in 3 der 6 eingeschlossenen Arbeiten gegeben. Informationen zu einem speziellen Entlass- und Versorgungsmanagement für MmkB und MmD waren in 5 von 6 Arbeiten enthalten.

Diskussion. Der Artikel illustriert bestehende Versorgungslücken von älteren $\mathrm{MmkB}$ an der Schnittstelle des Entlassmanagements und zeigt die Notwendigkeit neuer Versorgungsmodelle. Inwieweit diese strukturell, prozessual und systemisch in die Regelversorgung eingebettet und finanziert werden können, ist bislang offen und unerforscht.

\section{Schlüsselwörter}

Demenz · Kognitive Beeinträchtigung · Krankenhaus · Deutschland · Entlassungsmanagement

\section{Discharge management in German hospitals for cognitively impaired, older people-a scoping review}

\section{Abstract}

Background. In German hospitals approximately $40 \%$ of all patients over the age of 65 years are cognitively impaired (MmkB). After discharge from hospital it is particularly important for MmkB that the transition to domestic life is organized as seamlessly and as well-coordinated as possible.

Objective. The aim of the study was to determine the existing evidence on discharge management (EM) in MmkB and people with dementia (MmD). Furthermore, the study examined the necessity of an intersectoral concept for MmkB.

Material and methods. Based on a scoping review a total of 102 publications were identified, of which 6 articles were finally used for the evaluation.

Results. The article provides an overview of the current care of MmkB in acute care hospitals in Germany. General information on $E M$ in hospitals was given in three of the six publications included. Information on special discharge and care management for MmkB and $M m D$ was contained in five out of six papers.

Discussion. The article illustrates significant gaps in the hospital care for older MmkB, in particular at the interface of discharge management and demonstrates the need for new care models. To what extent these new care models can be structurally, procedurally and systemically embedded in the standard care and financed, is an open and unexplored question.

\section{Keywords}

Dementia · Cognitive impairment · Hospital · Germany · Discharge management 


\begin{tabular}{|c|c|c|}
\hline Autor (Jahr) & Titel & Quelle \\
\hline Nikolaus et al. (1992) & "Frühe Rehospitalisierung hochbetagter Patienten: Ursachen und Prävention" & {$[24]$} \\
\hline Angerhausen (2008) & $\begin{array}{l}\text { „Demenz - eine Nebendiagnose im Akutkrankenhaus oder mehr? Maßnahmen für eine bessere Versorgung } \\
\text { demenzkranker Patienten im Krankenhaus" }\end{array}$ & {$[2]$} \\
\hline Haude et al. (2009) & $\begin{array}{l}\text { "Treatment characteristics of patients with dementia: comparing two different psychiatric inpatient } \\
\text { settings" }\end{array}$ & [17] \\
\hline Bliemel et al. (2015) & $\begin{array}{l}\text { "Effect of preexisting cognitive impairment on in-patient treatment and discharge management among } \\
\text { elderly patients with hip fractures" }\end{array}$ & [6] \\
\hline $\begin{array}{l}\text { Von Renteln-Kruse et al. } \\
\text { (2015) }\end{array}$ & $\begin{array}{l}\text { "Geriatric patients with cognitive impairment: patient characteristics and treatment results on a specialized } \\
\text { ward" }\end{array}$ & [26] \\
\hline Zieschang et al. (2010) & $\begin{array}{l}\text { "Improving care for patients with dementia hospitalized for acute somatic illness in a specialized care unit: } \\
\text { a feasibility study" }\end{array}$ & [31] \\
\hline
\end{tabular}

\section{Patientenzentrierte Outcomes}

Patientenzentrierte Outcomes wurden über den funktionellen Status der Probanden (Barthel-Index), Mobilitätstest (TUG, Tinetti-Test), die Entlassmedikation und den Wohnort vor und nach dem Krankenhausaufenthalt gemessen. In den untersuchten Studien zeigte sich mehrheitlich eine Verbesserung der patientenzentrierten Outcomes, wenn ein Überleitungs- und/oder Konzept zum Umgang mit kognitiv beeinträchtigten Patienten während der Entlassung aus dem Krankenhaus zur Verfügung stand. Die Effekte eines Überleitungskonzeptes auf die patientenzentrierten Outcomes zeigt • Abb. 2.

\section{(Interventions-)Effekte}

Die relevanten Publikationen können nach Beobachtungs- und Interventionsstudien unterschieden werden. Während es sich bei den Studien von Haude et al. [17] und Renteln-Kruse et al. [26] um reine Beobachtungen mit Gegenüberstellung der patientenzentrierten Outcomes handelt, wird in der Studie von Zieschang et al. [31] eine Interventionsgruppe (mit Aufenthalt auf einer speziellen Demenzstation) mit Daten der Gesamtklinik aus der Routinebehandlung verglichen. Bliemel et al. [6] stellen den funktionalen Status von kognitiv beeinträchtigten $\mathrm{Pa}$ tienten mit einer Schenkelhalsfraktur in den Vordergrund und kristallisieren die Frührehabilitation als Lösungsansatz für diese spezielle Patientenklientel heraus. Zwei frühere Studien von 1992 [24] und 2008 [2] geben über die patientenzen- trierten Outcomes hinaus konkrete Kausalitätseffekte an. Während bei Nikolaus et al. [24] durch eine retrospektive Studie Kausalitätseffekte zur Verringerung der Rehospitalisierung aufgezeigt werden, werden im Rahmen eines ganzheitlichen Versorgungskonzeptes in der Arbeit von Angerhausen [2] konzeptionelle Effekte in den Dimensionen „Überleitung und Vernetzung“ und „medizinische Betreuung “ dargestellt. Unter anderem begleitet dabei eine Demenzbeauftragte die Patienten und deren Angehörige, schult das Personal entsprechend und unterstützt in herausfordernden Situationen im innerklinischen Setting. Weitere Orientierungshilfen im Krankenhaus und ein dicht am Alltag des Patienten strukturierter Tag (z. B. mit Berücksichtigung der Essensvorlieben etc.) helfen u. a. dabei, die Übergänge in der Versorgung möglichst reibungslos verlaufen zu lassen und Rehospitalisierungen zu verringern [2].

\section{Gemeinsamkeiten - Unterschiede}

Zwei der 6Studien stellen die Frührehabilitation als Lösungsansatz in den Vordergrund $[6,26]$. Die Studien von RentelnKruse et al. und Bliemel et al. sind im Jahre 2015 erschienen. Die früheren Studien $(2010,2009,2008,1992)$ fokussieren die Idee eines ganzheitlichen, intersektoralen Versorgungsarrangements für $\mathrm{MmkB}$ in einem Akutkrankenhaus. Nur Angerhausen (2008) spricht die Gestaltung der Räumlichkeiten auf einer Spezialstation für MmkB an [2]. Einzig in der Studie von Bliemel [6] wurden bereits perioperative Therapieziele (z. B. Verbesserung der Mo- bilität während des Krankenhausaufenthaltes) benannt. Nikolaus [24] stellt retrospektiv heraus, dass die häufigsten offenen Versorgungsbedarfe und Hauptrisiken für eine frühe Rehospitalisierung in den Bereichen der Medikamenteneinnahme, des Essverhaltens (Diäten etc.) und der Hilfsmittelbereitstellung bestehen.

\section{Innovative Ansätze}

Beide Studien aus 2015 berücksichtigen die Wohnsituation vor und nach einem akut-stationären Aufenthalt von $\mathrm{MmkB}$ $[6,26]$. Dass eine spezielle, demenzsensible Pflege - von den Autoren als Demenzpflege beschrieben - in den Krankenhäusern den funktionellen Status (ADL) der Patienten positiv beeinflusst und die institutionalisierte Pflege nach Entlassung in die Häuslichkeit reduzieren kann, zeigten die Studien von Haude et al. und Zieschang et al. [17, 31]. Die früheren Studien betonen im Rahmen ihrer Ganzheitlichkeitsbetrachtung, dass für MmkB in einem Akutkrankenhaus eher ein Überleitungs-, denn ein Entlassmanagement zur Verfügung stehen sollte und untergliedern ein sog. Versorgungsarrangement in die Dimensionen der „medizinischen Versorgung“ und „Überleitung und Vernetzung“ [2, 24]. Wegweisend ist dabei der verknüpfende, intersektorale Ansatz durch Fokussierung auf eine vernetzende Kommunikation der behandelnden und an der Patientenbetreuung und -versorgung teilnehmenden Professionen. 


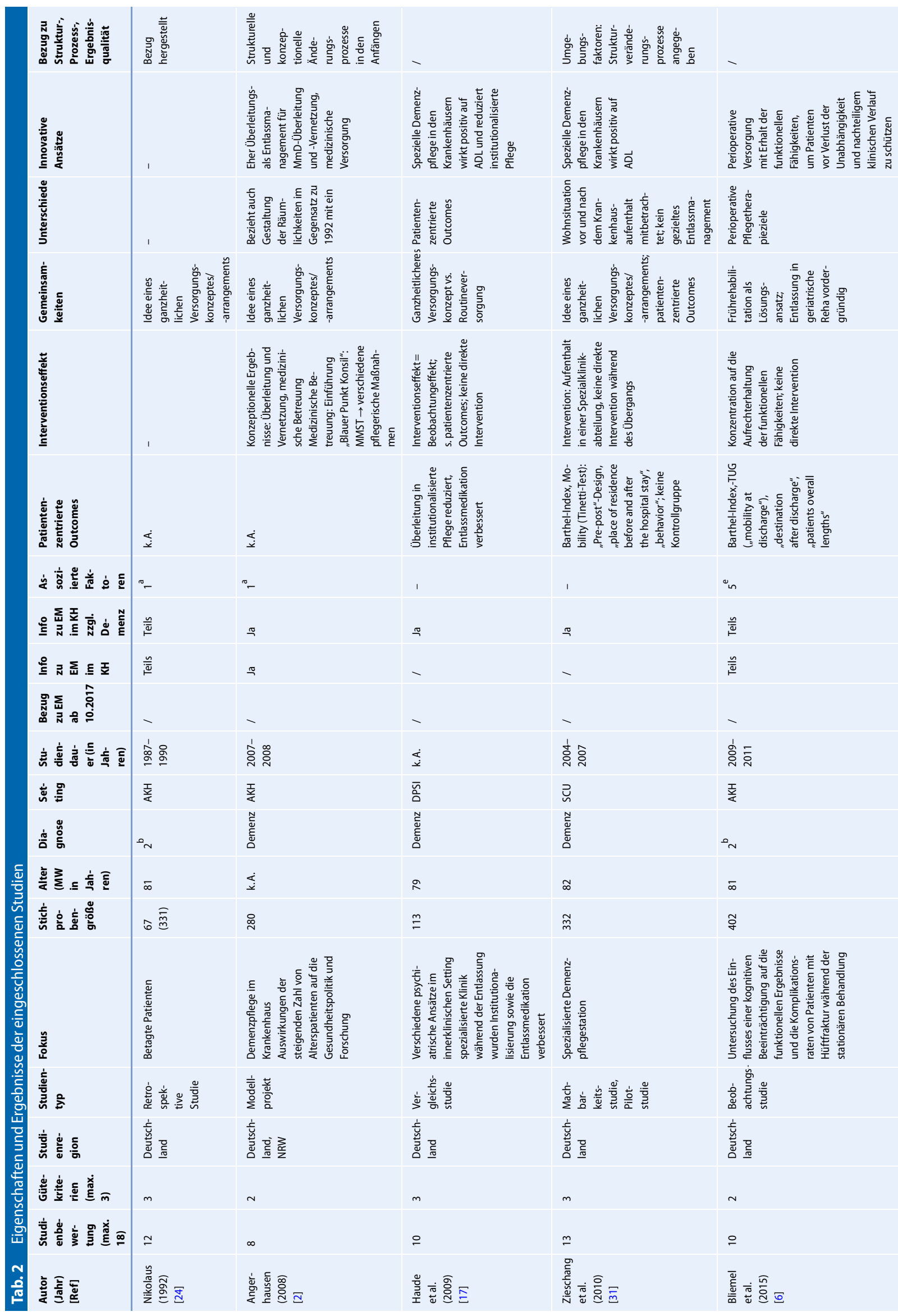




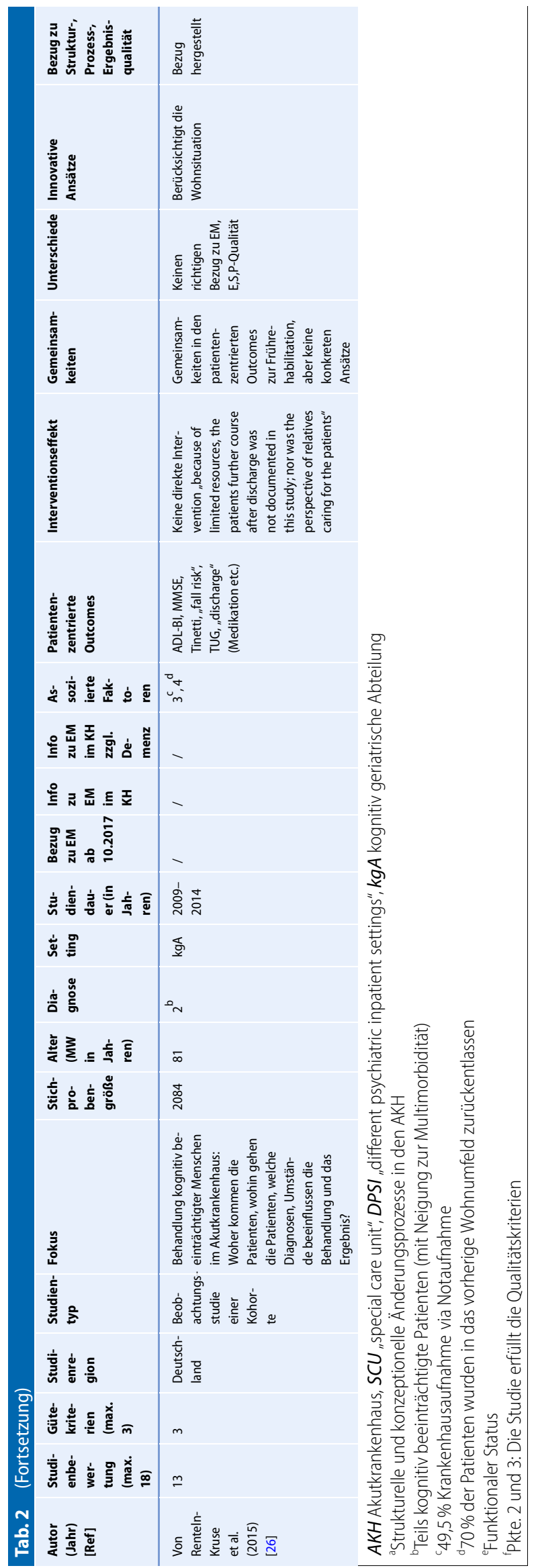

\section{Struktur-, Prozess- und Ergebnisqualität}

Im Verlauf des Abstract screening wurden Artikel analysiert und in die Übersichtsarbeit eingeschlossen, die Angaben zu Struktur-, Prozess- und Ergebnisqualität beinhalteten. Angaben dieser Art sind wichtige Indikatoren dafür, dass ein ganzheitliches Entlass-/ Überleitungsmanagement an der akutstationären Schnittstelle zurück in die Häuslichkeit der kognitiv beeinträchtigten Menschen perspektivisch in eine Art Regelversorgung übernommen werden kann. In 3 der 6 Studien wurden keine Angaben zu Struktur-, Prozess- und Ergebnisqualität gemacht. Angerhausen (2008) betonte, dass sich strukturelle und konzeptionelle Änderungsprozesse sich in den Anfängen befinden [2]; Zieschang bezog Umgebungsfaktoren im Rahmen der Strukturveränderungsprozesse unter Angabe unter dem Aspekt der Kosten ein [31]. Von Renteln-Kruse et al. stellen im Zusammenhang mit ihrer Studie in einem Zitat der WHO heraus, dass „[...] auch Verbesserungen in der Struktur erforderlich [sind]", die nicht nur die Logistik und Organisation der Pflege betreffen. „Diese Aufgaben liegen in der Verantwortung aller beteiligten Fachkräfte [... ]“ [26].

\section{Diskussion}

Zusammenfassend konnten kaum Publikationen, die das Entlassmanagement deutscher Krankenhäuser im Umgang mit kognitiv beeinträchtigten Menschen thematisieren und der internationalen, wissenschaftlichen Literatur zugänglich sind, gefunden werden. Untersuchungen $\mathrm{zu}$ Interventionsstudien, die ein reines Übergangsmanagement von $\mathrm{MmkB}$ an der Schnittstelle Akutkrankenhaus - Häuslichkeit eruieren, fanden nicht statt. Patientenzentrierte Outcomes an der Schnittstelle Klinik - Häuslichkeit geben Anhaltspunkte für Interventionsmöglichkeiten. Diese bieten jedoch kein evidenzbasiertes Feedback, welches Entscheidungsträger zu einem Umdenken in der Versorgungslandschaft und -qualität an der Schnittstelle Akutkrankenhaus - Häuslichkeit von Menschen 


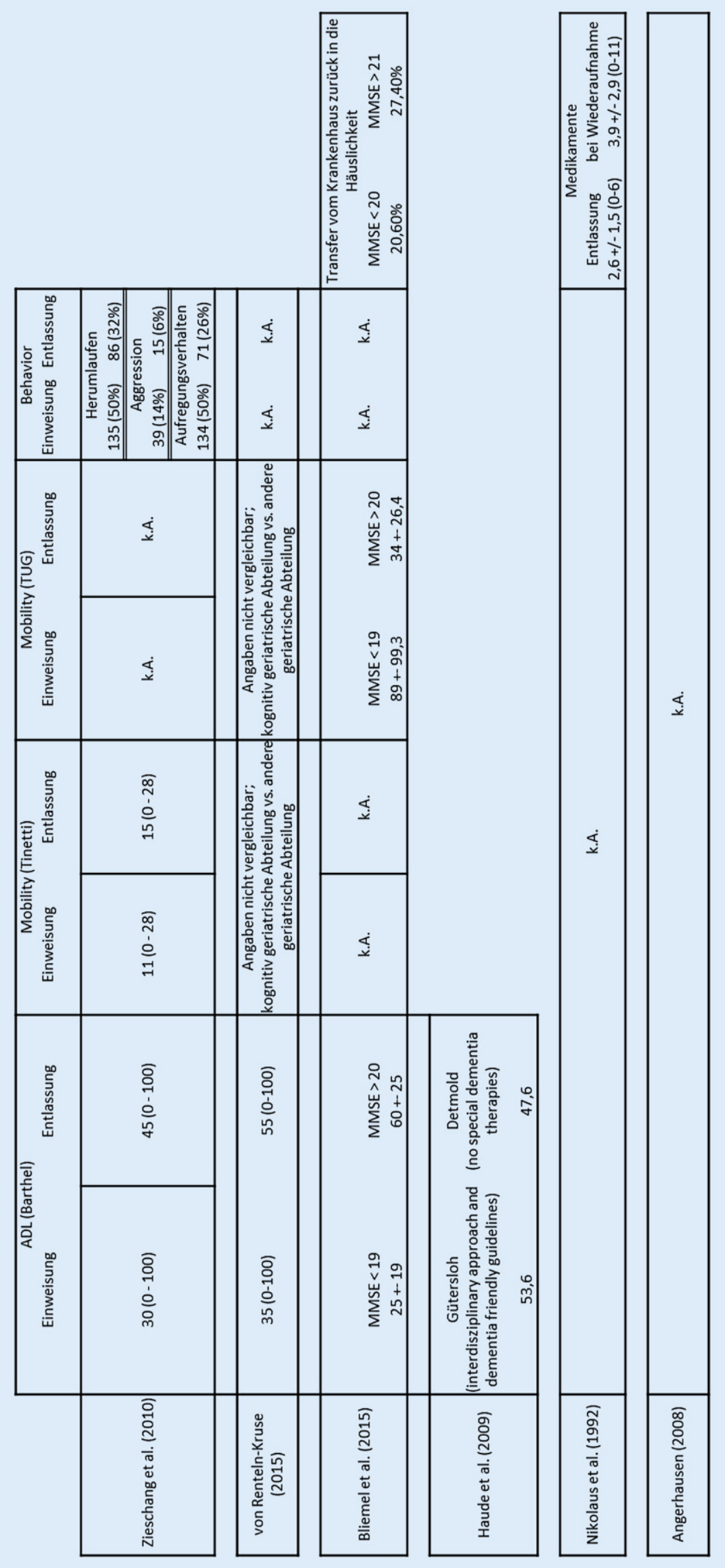

Abb. $2 \varangle$ Patientenzentrierte Outcomes eingeschlossener Studien. Barthel-ADL-Score: 0 (geringe Alltagskompetenz) - 100 (ausgezeichnete Alltagskompetenz) | Tinetti-Score: 0 bis 9 Punkte: Mobilität massiv eingeschränkt, Sturzrisiko massiv erhöht, 10 bis 14 Punkte: Mobilität mäBig eingeschränkt, Sturzrisiko deutlich erhöht, 15 bis 19 Punkte: Mobilität leicht eingeschränkt, Sturzrisiko erhöht, 20 bis 27 Punkte: Mobilität leicht eingeschränkt, Sturzrisiko evtl. erhöht, 28 Punkte: maximale Punktzahl: kein Hinweis auf Gang- oder Gleichgewichtsstörung

mit kognitiv beeinträchtigten Menschen leiten könnte. Diese sind jedoch wichtig, weil z.B. in den letzten Jahren in deutschen Krankenhäusern zahlreiche geriatrische Fachabteilungen gegründet wurden [22]. Die Altersmedizin verfügt mittlerweile über die zweitgrößte Anzahl von spezialisierten internistischen Betten in deutschen Krankenhäusern (Stand 09.2019). Die Studien, die dieser Analyse zugrunde liegen, geben Hinweise, dass „die frühzeitige und kontinuierliche Einbindung geriatrischer Kompetenz in die Behandlungsabläufe die Qualität der Versorgung hochbetagter, multimorbider Patienten steigern" kann; inwiefern hiervon ein Entlassmanagement profitiert, bedarf weiterer, zielgerichteter Untersuchungen (www.dggeriatrie.de, Stand 09.2019 [32]).

Es ist jedoch unklar, ob das Entlassmanagement kognitiv beeinträchtigter Menschen durch das Vorhandensein oder die Etablierung einer geriatrischen Abteilung in einem Akutkrankenhaus bereits ausreichend verbessert wird. Neben der Gründung von Fachabteilungen wird aber auch zunehmend konzeptionell im Akutkrankenhaus den Bedarfen der MmkB Rechnung getragen. Unter dem Begriff des demenzsensiblen Krankenhauses finden sich Bestrebungen und Beispiele, wie ein multiprofessionelles Versorgungsmanagement umgesetzt werden kann $[1,20]$.

\section{Limitationen}

Bei diesem Scoping Review handelt es sich um eine Übersichtsarbeit, in die Publikationen der internationalen, wissenschaftlichen Literaturdatenbank PubMed eingingen. Es wurden nur Artikel heran- 
gezogen, dessen Erhebungsort die Bundesrepublik Deutschland war.

Die Arbeit ist durch eine hohe externe Validität unter Berücksichtigung und der Prüfung einschlägiger Qualitätskriterien gekennzeichnet.

\section{Einschränkungen, Heraus- forderungen und zukünftige Forschung}

Es gibt Hinweise, dass das sektorenübergreifende Entlassmanagement im Allgemeinkrankenhaus als Teil der akutstationären Versorgung eine breite Akzeptanz gefunden habe [16], eine solide empirische Evidenz fehlt jedoch. Welche Konzepte eine bessere und effizientere und auf das Behandlungsergebnis kognitiv beeinträchtigter Patienten noch stärker patientenzentrierte Versorgung fördern, bleibt unklar. Es existieren Veröffentlichungen um den Themenkomplex „Entlassmanagement, Überleitungsmanagement, Pflegeüberleitung " $[3,4,7$, 9, 13]. Ein effektives (in diesem Sinne demenzsensibles) Entlassmanagement erfordert ein komplexes, multiprofessionelles (Versorgungs-)Management, in das zahlreiche Variablen hineinspielen, die meist keine statischen, sondern sich gegenseitig beeinflussende, dynamische Faktoren sind.

\section{Schlussfolgerungen}

In den untersuchten Studien zeigt sich, dass frühe Rehospitalisierungen und ungeplante Institutionalisierungen sich häufig vermeiden lassen; so wird geschätzt, dass 55,5\% der Rehospitalisierungen, die durch eine inadäquate häusliche Versorgung bedingt waren, hätten vermieden werden können, wenn vor der Entlassung aus der Klinik eine individuelle [geriatrische] Gesamteinschätzung vorgenommen und das weitere Vorgehen sorgfältig geplant und aktiv vorbereitet wird [21]. Darüber hinaus wurde deutlich, dass

A. evidenzbasierte Konzepte zum Ent-

lassmanagement bei kognitiv beeinträchtigten Patienten fehlen; diese treffen nach der Krankenhausentlassung in ihrer Häuslichkeit oftmals noch auf eine unzureichende häusliche Betreuung.

B. auf eine Demenz/kognitive Beeinträchtigung zugeschnittene Behandlungsprogramme positiven Einfluss auf die patientenzentrierten Outcomes nehmen. Es wird angenommen, dass beispielsweise Komplikationen aus einer raschen Progression der Grundkrankheit sowie eine oftmals vorliegende Noncompliance bei der Medikamenteneinnahme oder der häuslichen Ernährung resultieren [21].

C. davon auszugehen ist, dass sich mit einer umfassenden Entlassplanung bzw. einer Übergangsbetreuung nach der Entlassung, einer ausführlichen schriftlichen Dokumentation an die versorgenden Ärzte und die Unterstützung durch ambulante Dienste Rehospitalisierungen und ungeplante Heimeinweisungen reduzieren lassen.

\section{Fazit für die Praxis}

- Auf das Krankenhaus beschränkte Konzepte zeigen positive Auswirkungen auf die patientenzentrierten Outcomes.

- Wichtige Komponenten sind: ein positiver Einfluss auf den funktionellen Status (z. B. ADL) der Patienten, eine umfassende Entlassplanung/ Übergangsbetreuung, ausführliche, schriftliche Dokumentationen an die (weiter-)versorgenden Ärzte und Unterstützungsangebote durch ambulante Dienste.

- Lösungsansätze zum Management der Überleitung vom Krankenhaus zurück in die Häuslichkeit von Menschen mit kognitiven Beeinträchtigungen sollten in der Praxis erprobt, evaluiert und implementiert werden.

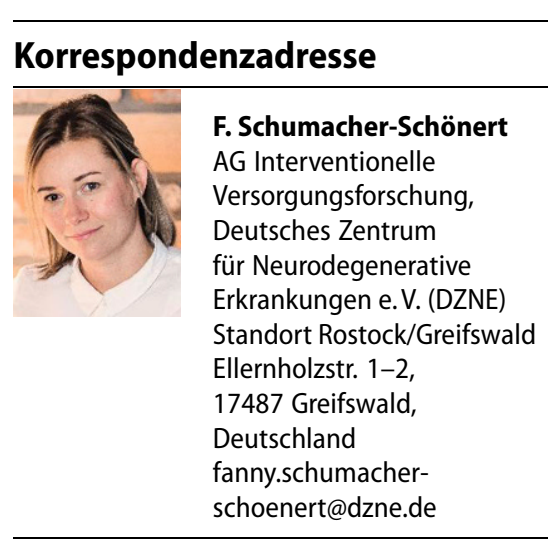

Funding. Open Access funding provided by Projekt DEAL.

\section{Einhaltung ethischer Richtlinien}

Interessenkonflikt. F. Schumacher-Schönert, D. Wucherer, A. Nikelski, S. Kreisel, H.C. Vollmar, W. Hoffmann und J.R. Thyrian geben an, dass kein Interessenkonflikt besteht.

Für diesen Beitrag wurden von den Autoren keine Studien an Menschen oder Tieren durchgeführt. Für die aufgeführten Studien gelten die jeweils dort angegebenen ethischen Richtlinien.

Open Access. Dieser Artikel wird unter der Creative Commons Namensnennung 4.0 International Lizenz veröffentlicht, welche die Nutzung, Vervielfältigung Bearbeitung, Verbreitung und Wiedergabe in jeglichem Medium und Format erlaubt, sofern Sie den/die ursprünglichen Autor(en) und die Quelle ordnungsgemäß nennen, einen Link zur Creative Commons Lizenz beifügen und angeben, ob Änderungen vorgenommen wurden.

Die in diesem Artikel enthaltenen Bilder und sonstiges Drittmaterial unterliegen ebenfalls der genannten Creative Commons Lizenz, sofern sich aus der Abbildungslegende nichts anderes ergibt. Sofern das betreffende Material nicht unter der genannten Creative Commons Lizenz steht und die betreffende Handlung nicht nach gesetzlichen Vorschriften erlaubt ist, ist für die oben aufgeführten Weiterverwendungen des Materials die Einwilligung des jeweiligen Rechteinhabers einzuholen.

Weitere Details zur Lizenz entnehmen Sie bitte der Lizenzinformation auf http://creativecommons.org/ licenses/by/4.0/deed.de.

\section{Literatur}

\section{Verwendete Literatur}

1. Amadori K, Augustin U, Bauer J, Böckel H, Brune M, Dahlem C, Donders P, Engel S, Feddersen E, Glarcher M (2019) Das demenzsensible Krankenhaus: Grundlagen und Praxis einer patientenorientier- 
ten Betreuung und Versorgung. Kohlhammer, Stuttgart

2. Angerhausen S (2008) Demenz - eine $\mathrm{Ne}-$ bendiagnose im Akutkrankenhaus oder mehr? Maßnahmen für eine bessere Versorgung demenzkranker Patienten im Krankenhaus. Z Gerontol Geriat 41:460-466

3. Ballsieper K, Lemm U, Reibnitz C (2012) Überleitungsmanagement: Praxisleitfaden für stationäre Gesundheitseinrichtungen. Springer, Berlin, Heidelberg

4. Ballsieper K, Lemm U, von Reibnitz C (2012) Grundlagen des Überleitungsmanagements. Überleitungsmanagement. Springer, Berlin, Heidelberg, $\mathrm{S} 1-52$

5. Bickel $H$, Hendlmeier I, Heßler JB, Junge MN, Leonhardt-Achilles S, Weber J, Schäufele M (2018) Prävalenz von Demenz und kognitiver Beeinträchtigung in Krankenhäusern. Dtsch Arztebl Int 115:733-740

6. Bliemel C, Lechler P, Oberkircher L, Colcuc C, Balzer-Geldsetzer M, Dodel R, Ruchholtz S, Buecking B (2015) Effect of preexisting cognitive impairmenton in-patient treatment and discharge management among elderly patients with hip fractures. Dement Geriatr Cogn Disord 40:33-43

7. Borgwardt M, Hammermüller S (2017) Ein Höchstmaß an Normalität. intensiv 25:304-307

8. Bracke J, Güttner-Scarfone C (2017) Entlassmanagementnach §39SGBV-eineHerausforderung Gesundheits- und Sozialpolitik 71:44-47

9. Bühler E (2006) Überleitungsmanagement und integrierte Versorgung: Brücke zwischen Krankenhaus und nachstationärer Versorgung. Kohlhammer, Stuttgart

10. Callahan CM, Tu W, Unroe KT, LaMantia MA, Stump TE, Clark DO (2015) Transitions in care in a nationally representative sample of older Americans with dementia. J Am Geriatr Soc 63:1495-1502

11. Chenoweth L, Kable A, Pond D (2015) Research in hospital discharge procedures addresses gaps in care continuity in the community, but leaves gaping holes for people with dementia: a review of the literature. Australas J Ageing 34:9-14

12. Daiello LA, Gardner R, Epstein-Lubow G, Butterfield K, Gravenstein S (2014) Association of dementia with early rehospitalization among medicare beneficiaries. Arch Gerontol Geriatr 59:162-168

13. Deimel D, Müller M-L (2012) Entlassmanagement: Vernetztes Handeln durch Patientenkoordination. Thieme, Stuttgart

14. Dewing J, Dijk S (2016) What is the current state of care for older people with dementia in general hospitals? A literature review. Dementia 15:106-124

15. von Elm E, Schreiber G, Haupt CC (2019) Methodische Anleitung für Scoping Reviews (JBIMethodologie). Z Evid Fortbild Qual Gesundhwes 143:1-7

16. Gürkan I (2017) Entlassmanagement: Chance für mehr Effizienz in den Krankenhäusern und bessere Versorgungsqualität. Gesundh Ökon Qual Manag 22:171-172

17. Haude V, Lüdeke M, Dohse $H$, Reiswig S, Liebler A, Assion H-J, Basilowski M, Börner I (2009) Treatment characteristics of patients with dementia: comparing two different psychiatric inpatient settings. Am J Alzheimers Dis Other Demen 24:228-233

18. JacobsK, Kuhlmey A, GreßS, Klauber J, Schwinger A (2016) Pflege-Report 2017. Schwerpunkt: Die
Versorgung der Pflegebedürftigen. Schattauer, Stuttgart

19. Kable A, Chenoweth L, Pond D, Hullick C (2015) Health professional perspectives on systems failures in transitional care for patients with dementia and their carers: a qualitative descriptive study. BMC Health Serv Res 15:567

20. Kirchen-Peters S, Krupp E (2019) Praxisleitfaden zum Aufbau demenzsensibler Krankenhäuser. Robert Bosch Stiftung, Stuttgart

21. Langer J, Ewers M (2013) "It's not the old life that we live anymore..."-counselling of relatives in nurse discharge planning. Pflege 26:311-320

22. Meier-Baumgartner H-P (2001) Geriatrie - Einbettung in die Versorgungslandschaft der Bundesrepublik Deutschland. Z Gerontol Geriat 34:1001-1009

23. Moyle W, Olorenshaw R, Wallis M, Borbasi S (2008) Best practice for the management of older people with dementia in the acute care setting: a review of the literature. Int J Older People Nurs 3:121-130

24. Nikolaus T, Specht-Leible N, Kruse W, Oster P, Schlierf G (1992) Frühe Rehospitalisierung hochbetagter Patienten: Ursachen und Prävention. Dtsch Med Wochenschr 117:403-407

25. Pinkert C, Holle B (2012) People with dementia in acute hospitals. Literature review of prevalence and reasons for hospital admission. Z Gerontol Geriat 45:728-734

26. von Renteln-Kruse W, Neumann L, Klugmann B, Liebetrau A, Golgert S, Dapp U, Frilling B (2015) Geriatric patients with cognitive impairment: patient characteristics and treatment results on a specialized ward. Dtsch Arztebl Int 112:103

27. Ritschl V, Mosor E, Ritschl U, Stamm T, Becker H, Sturma A (2016) Themenfindung und Recherche. Wissenschaftliches Arbeiten und Schreiben. Springer, Berlin, Heidelberg, S269-279

28. Schmidt S (2016) Expertenstandard Entlassungsmanagement in der Pflege. Expertenstandards in der Pflege - eine Gebrauchsanleitung. Springer, Berlin, Heidelberg, S41-58

29. van de Vorst IE, Vaartjes I, Geerlings MI, Bots ML, Koek HL (2015) Prognosis of patients with dementia: results from a prospective nationwide registry linkage study in the Netherlands. BMJ Open 5:e8897

30. Wingenfeld K, Joosten M, Müller C, Ollendiek I (2007) Pflegeüberleitung in Nordrhein-Westfalen: Patientenstruktur und Ergebnisqualität. Universität Bielefeld, Bielefeld

31. Zieschang T, Dutzi I, Müller E, Hestermann U, Grünendahl K, Braun AK, Hüger D, Kopf D, SpechtLeible N, Oster P (2010) Improving care for patients with dementia hospitalized for acute somatic illness in a specialized care unit: a feasibility study. Int Psychogeriatr 22:139-146

32. Deutsche Gesellschaft für Geriatrie (DGG) (2019) Was ist Geriatrie? https://www.dggeriatrie.de/ nachwuchs/91-was-ist-geriatrie.html. Zugegriffen: 12. Sept. 2019

\section{Weiterführende Literatur}

33. Alzheimer's Disease International (ADI) (2016) World Alzheimer report 2016. https://www.alz. co.uk/research/WorldAlzheimerReport2016.pdf. Zugegriffen: 13. März 2019

34. Deutsche Alzheimer Gesellschaft e.V. (2018) Die Häufigkeit von Demenzerkrankungen. https://www.deutsche-alzheimer.de/fileadmin/ alz/pdf/factsheets/infoblatt1_haeufigkeit
demenzerkrankungen_dalzg.pdf.Zugegriffen: 13 . März 2019

35. Deutsches Netzwerk für Qualitätsentwicklung in der Pflege (DNQP) (2009) Expertenstandard Entlassungsmanagement in der Pflege - 1. Aktualisierung 2009. Schriftenreihe des Deutschen Netzwerkes für Qualitätsentwicklung in derPflege. Osnabrück 